\title{
An interdimensional correlation framework for real-time estimation of six degree of freedom target motion using a single $x$-ray imager during radiotherapy
}

\section{T Nguyen', J Bertholet ${ }^{3}$, J-H Kim¹, R O'Brien', J T Booth², P R Poulsen', P J Keall ${ }^{1}$}

${ }^{1}$ Radiation Physics Laboratory, Sydney Medical School, The University of Sydney, Australia ${ }^{2}$ Northern Sydney Cancer Centre, Royal North Shore Hospital, Australia

${ }^{3}$ Department of Oncology, Aarhus University Hospital, Denmark

E-mail: d.nguyen@sydney.edu.au

\begin{abstract}
.
Purpose: Increasing evidence suggests that intrafraction tumour motion monitoring needs to include both 3D translations and 3D rotations. Presently, methods to estimate the rotation motion require the 3D translation of the target to be known first. However, ideally, translation and rotation should be estimated concurrently. We present the first method to directly estimate six-degree-offreedom $(6 \mathrm{DoF})$ motion from the target's projection on a single rotating $\mathrm{x}$-ray imager in real-time.
\end{abstract}

Methods: This novel method is based on the linear correlations between the superior-inferior translations and the motion in the other five degrees-of-freedom. The accuracy of the method was evaluated in silico with 81 liver tumour motion traces from 19 patients with three implanted markers. The ground-truth motion was estimated using the current gold standard method where each marker's 3D position was first estimated using a Gaussian probability method, and the $6 \mathrm{DoF}$ motion was then estimated from the 3D positions using an iterative method.

The 3D position of each marker was projected onto a gantry-mounted imager with an imaging rate of $11 \mathrm{~Hz}$. After an initial $110^{\circ}$ gantry rotation (200 images), a correlation model between superior-inferior translations and the five other DoFs was built using a least square method. The correlation model was then updated after each subsequent frame to estimate 6DoF motion in real-time.

Results: The proposed algorithm had an accuracy ( \pm precision) of $-0.03 \pm 0.32 \mathrm{~mm}$, $-0.01 \pm 0.13 \mathrm{~mm}$ and $0.03 \pm 0.52 \mathrm{~mm}$ for translations in the left-right (LR), superiorinferior (SI) and anterior-posterior (AP) directions respectively; and, $0.07 \pm 1.18^{\circ}$, $0.07 \pm 1.00^{\circ}$ and $0.06 \pm 1.32^{\circ}$ for rotations around the LR, SI and AP axes respectively on the dataset.

Conclusion: The first method to directly estimate real-time 6DoF target motion from segmented marker positions on a $2 \mathrm{D}$ imager was devised. The algorithm was evaluated using 81 motion traces from 19 liver patients and found to have sub$\mathrm{mm}$ and sub-degree accuracy.

Submit to: Phys. Med. Biol.

Keywords: intrafraction tumour motion, real-time motion monitoring, six degrees-of-freedom, tumour rotation, interdimensional correlation, radiotherapy. 


\section{Introduction}

In current radiation therapy, image guided radiation therapy (IGRT) is routinely applied at the start of treatment to align the target with its planned position. However, tumours in the thorax, abdomen and pelvis are not static during treatment. Hence, methods to monitor tumour motion during treatment are highly desirable, even more so with dose escalation and hypofractionation.

A number of different intrafraction real-time guidance methods have been used during prostate cancer treatments. Systems such as CyberKnife (Accuray, Sunnyvale, CA) and the real-time tracking radiotherapy (RTRT) system use real-time kilovoltage $(\mathrm{kV})$ images from two (CyberKnife) or four (RTRT system) orthogonal room-mounted imagers to track the prostate position based on segmented positions of implanted fiducial markers (King et al., 2009, Kitamura et al., 2002, Sazawa et al., 2009, Shimizu et al., 2000, Shirato et al., 2003, Shirato et al., 2000). Calypso (Varian, Palo Alto, CA) (Kupelian et al., 2007) and RayPilot (Micropos, Gothenburg, Sweden) (Castellanos et al., 2012) utilise implanted electromagnetic transponders, transmitting positional signals to an external receiver. Emerging real-time guidance technologies include ultrasonography (Ballhausen et al., 2015) and integrated magnetic resonance imaging (MRI)-radiation therapy systems (Fallone et al., 2009, Raaymakers et al., 2009) . Common to all these methods is the need for additional dedicated and typically expensive equipment to perform the real-time guidance.

Ideally, real-time image guidance would be performed using a standard linear accelerator (linac) without relying on additional hardware. To this end, a number of algorithms have been proposed for the purpose of estimating the target's position in 3D based on its location on a $2 \mathrm{D}$ image, which can be acquired using a linear accelerator gantry mounted kilovoltage $(\mathrm{kV}) \mathrm{x}$-ray imager system. An apparent advantage of utilising the $\mathrm{kV}$ imager is that: most modern linear accelerator have a $\mathrm{kV}$ imager, mounted orthogonally to the treatment beam. However, as the target position on the $\mathrm{kV}$ imager only contains $2 \mathrm{D}$ information, a $2 \mathrm{D} \rightarrow 3 \mathrm{D}$ target position conversion is often required. The sparse information renders the problem of solving for the target's 3D position ill-posed, hence, some a priori knowledge or assumption is usually required.

\subsection{The problem of $2 D \rightarrow 3 D$ estimation}

30 Poulsen et al. (2008b) proposed a Maximum Likelihood Estimation (MLE) algorithm to estimate the target's 3D position assuming a Gaussian distribution, which can be built after a learning arc. This solution has been clinically implemented as the Kilovoltage Intrafraction Monitoring (KIM) system, and is currently being trialled for real-time tumour motion guidance in a pilot clinical trial and a multi-centre clinical trial (Keall et al., 2016, Nguyen et al., 2017). Recently, a Bayesian method to estimate the proper distribution of the target was also proposed (Li et al., 2011), which does not assume Gaussian distribution and hence may be more accurate in estimating the target's respiratory motion as the thoracic tumour motions are complicated and can be asymmetrical as well as hysteric.

Other $2 \mathrm{D} \rightarrow 3 \mathrm{D}$ methods that do not follow the probabilistic approach have also been proposed. For Cone Beam CT (CBCT) trajectories reconstruction, the positions of the target in 3D can be estimated using phase-binning, and linear interpolation, assuming the target's position in 3D do not change much within each respiratory bin (Park et al., 2012, Becker et al., 2010). However, this method requires all projections to be collected before $2 \mathrm{D} \rightarrow 3 \mathrm{D}$ estimation and hence, is not suitable for real-time target positional estimation during treatment. 
A different approach for $2 \mathrm{D} \rightarrow 3 \mathrm{D}$ estimation is to make use of interdimensional correlation (IDC), which works for two reasons: (1) thoracic tumour motion in the Anterior-Posterior (AP) and Left-Right (LR) are correlated to its motion in the Superior-Inferior (SI) direction; and (2) as the gantry rotates around the patient, the SI position of the tumour is always visible on the $\mathrm{kV}$ images. Poulsen et al. (2008) implicitly incorporated IDC into their MLE of a Gaussian distribution. Other authors have shown that IDC can be used exclusively for 2D $\rightarrow 3 \mathrm{D}$ estimation, i.e without probability estimation, including the works of Cho et al. (2012) and Chung et al. (2016). An advantage of this method is that it can be extended to include the signal from an external surrogate, which is also correlated with the 3D motion of the target. Additionally, the linear model of IDC could be expanded to include state-augmentation to account for hysteresis in thoracic tumour motions (Ruan et al., 2008).

\subsection{The problem of $2 D \rightarrow 6 D$ estimation}

Increasing evidence suggests that intrafractional tumour motion corrections should be applied for both tumour translations and tumour rotations (Amro et al., 2013, Rijkhorst et al., 2009, Wu et al., 2011). Retrospective post-treatment calculation of tumour rotations have shown that the rotations could be significant for both prostate and lung tumours (Amro et al., 2013, Aubry et al., 2003). Dosimetrically, uncorrected prostate rotations of $15^{\circ}$ can result in a $12 \%$ under dose to the tumour (Rijkhorst et al., 2009). Tumour rotation estimation using KIM has been developed using the iterative closest point (ICP) algorithm (Tehrani et al., 2013). This method has been used retrospectively to quantify translational and rotational motion for prostate and lung cancers (Huang et al., 2015) and liver cancer (Bertholet et al., 2016). A disadvantage of this method is that the rotational motions are solved after the translational motions as the ICP algorithm requires target positions in 3D.

In this work, we propose a novel method that expands upon the 2D $\rightarrow 3 \mathrm{D}$ IDC formalism to solve for six degrees of freedom in one step. The rationale behind this novel method is supported by the works of Huang et al. (2015) and Bertholet et al. (2016) that revealed strong correlations between the rotational motions and the superior-inferior translational motion in both lung and liver tumours. The accuracy and precision of the proposed algorithm are evaluated in silico with patients' tumour liver traces during CBCT scans prior to radiotherapy as described in (Bertholet et al., 2016).

This paper describes a direct method for estimating real-time 6DoF target motion from the target's positions on a 2D imager that is mounted on the gantry of a standard linac. Previous efforts to calculate $6 \mathrm{DoF}$ motion from the target's $2 \mathrm{D}$ positions were done in two steps: (i) estimating the $3 \mathrm{D}$ position of each point of the target; and (ii) calculating the $6 \mathrm{DoF}$ motion by iteratively minimising the sum of squared differences in estimated and measured 3D positions of the points of the target (Tehrani et al., 2013, Huang et al., 2015, Bertholet et al., 2016). The implication of the prior work is that if errors occurs in the first step, the rotation estimation can become erroneous as well. The presented algorithm can be used to estimate 6 DoF tumour motion affected by the breathing motion from $2 \mathrm{D} \mathrm{kV}$ images in real-time. The method is thus applicable for use on standard-equipped modern linacs. 


\section{Methods}

In this section, we first describe the formalism of a novel method for estimate 6DoF motion form 2D projection of a target using the least square method. Then, we describe the VMAT simulation used to comprehensively evaluate the proposed algorithm, based on the patients' liver data, acquired in a clinical trial at the Aarhus University Hospital. Throughout this paper, we use the IEC 61217 coordinate system to describe the patient's motion relative to the treatment beam.

\subsection{The $2 D \rightarrow 6 D-I D C$ formalism}

\subsubsection{The forward problem: $6 D \rightarrow 2 D$}

In order to find a unique solution for the $6 \mathrm{DoF}$ motion of a target provided its projection in $2 \mathrm{D}$ is an inverse problem. To facilitate the description of this inverse problem, it helps to first describe the forward problem: If we know the target's $6 \mathrm{DoF}$ motions with respect to a reference, what is the projection of the object on a rotating $\mathrm{kV}$ imager?

In the Euclidean coordinates system, the rotational and translational position of a target $\boldsymbol{M}$ at time $\boldsymbol{t}$ with respect to a referenced position $\boldsymbol{M}_{\boldsymbol{r e f}}$ is defined as:

$$
\left(\begin{array}{l}
x \\
y \\
z
\end{array}\right)=R \cdot\left(\begin{array}{l}
x_{\text {ref }} \\
y_{\text {ref }} \\
z_{\text {ref }}
\end{array}\right)+\left(\begin{array}{l}
\operatorname{Tr}_{x} \\
\operatorname{Tr}_{y} \\
\operatorname{Tr}_{z}
\end{array}\right)
$$

where $R$ is the rotational matrix

$$
\boldsymbol{R}=\boldsymbol{R}_{\boldsymbol{x}} \boldsymbol{R}_{\boldsymbol{y}} \boldsymbol{R}_{\mathrm{z}}=
$$

$$
\left[\begin{array}{ccc}
\cos \beta \cos \gamma & -\cos \beta \sin \gamma & \sin \beta \\
\cos \alpha \sin \gamma+\sin \alpha \sin \beta \cos \gamma & \cos \alpha \cos \gamma-\sin \alpha \sin \beta \sin \gamma & -\sin \alpha \cos \beta \\
\sin \alpha \sin \gamma-\cos \alpha \sin \beta \cos \gamma & \sin \alpha \cos \gamma+\cos \alpha \sin \beta \sin \gamma & \cos \alpha \cos \beta
\end{array}\right]
$$

where $(\alpha, \beta, \gamma)$ are the angles describing the rotation of the object around the axes $x, y$ and $z$,

20 respectively. Note that the vector $\boldsymbol{T r}=\left(\begin{array}{l}\boldsymbol{T} \boldsymbol{r}_{\boldsymbol{x}} \\ \boldsymbol{T} \boldsymbol{r}_{\boldsymbol{y}} \\ \boldsymbol{T} \boldsymbol{r}_{z}\end{array}\right)$ is merely a mathematical by-product of the rotation equation to accurately relate a $3 \mathrm{D}$ object with coordinates $(\boldsymbol{x}, \boldsymbol{y}, \boldsymbol{z})$ with its referenced coordinates $\left(\boldsymbol{x}_{\boldsymbol{r e f}}, \boldsymbol{y}_{\boldsymbol{r e f}}, \boldsymbol{z}_{\boldsymbol{r e f}}\right)$. The vector $\boldsymbol{T r}$ on its own does not provide the translational motion information. The real translational vector is defined as simply a vector difference between the current centroid of the object and its referenced centroid coordinates 


$$
T=\bar{M}_{t}-\bar{M}_{r e f}=\left(\begin{array}{l}
T_{x} \\
T_{y} \\
T_{z}
\end{array}\right)=\left(\begin{array}{l}
\bar{x} \\
\bar{y} \\
\bar{z}
\end{array}\right)-\left(\begin{array}{l}
\bar{x}_{r e f} \\
\bar{y}_{r e f} \\
\bar{z}_{r e f}
\end{array}\right)
$$

Given the object $\boldsymbol{M}$ with the 3D coordinates $(\boldsymbol{x}, \boldsymbol{y}, \boldsymbol{z})$, we can find its projected position $\left(\boldsymbol{x}_{\boldsymbol{p}}, \boldsymbol{y}_{\boldsymbol{p}}\right)$ on the $\mathrm{kV}$ imager when the linac gantry is at a certain angle $\boldsymbol{\theta}$ with the following projection equation:

$$
P(x, y, z \mid \theta)=\left(\begin{array}{l}
x_{p} \\
y_{p}
\end{array}\right)(\theta)=\frac{\operatorname{sID}}{\operatorname{sAD}-(x \cdot \cos \theta+z \cdot \sin \theta)}\left(\begin{array}{c}
x \cdot \sin \theta-z \cdot \cos \theta \\
y
\end{array}\right)
$$

where SID is the source-to-imager distance and SAD is the source-to-axis distance, i.e. the distance between the $\mathrm{kV} \mathrm{X}$-ray source to the radiation isocentre.

Thus, from equations (1) and (2), we can determine the position of aa target projected onto the $\mathrm{kV}$ imager if the rotational matrix $\boldsymbol{R}$ and the vector $\boldsymbol{T r}$ are known. The reference position $\boldsymbol{M}_{\boldsymbol{r} \boldsymbol{e} \text { f }}$ in the context of external beam radiotherapy can be determined as the tumour position in the planning CT.

\subsubsection{The inverse problem: $2 D \rightarrow 6 D$}

Conversely, to solve for the matrix $\boldsymbol{R}$ and the vector $\boldsymbol{T r}$ given only the projected positions $\left(\begin{array}{l}\boldsymbol{x}_{\boldsymbol{p}} \\ \boldsymbol{y}_{\boldsymbol{p}}\end{array}\right)$ of the target is an ill-posed problem. However, given three or more points in the target $\boldsymbol{M}$, a solution can be found numerically, providing a priori knowledge. In this paper, our prior is that there is a linear correlation between the translational and rotational components of the object's motion (Bertholet et al., 2016, Huang et al., 2015). Additionally, we also assume that the target moves rigidly, without any deformations.

If the translational and rotational components of the target's motions are linearly correlated, the following equation can be assumed:

$$
\left(\begin{array}{c}
\widehat{T r}_{x} \\
\widehat{T r}_{y} \\
\widehat{T r}_{z} \\
\widehat{\alpha} \\
\widehat{\beta} \\
\widehat{\gamma}
\end{array}\right)(t)=\left(\begin{array}{l}
a_{x} \\
a_{y} \\
a_{z} \\
a_{\alpha} \\
a_{\beta} \\
a_{\gamma}
\end{array}\right) \cdot y(t)+\left(\begin{array}{l}
b_{x} \\
b_{y} \\
b_{z} \\
b_{\alpha} \\
b_{\beta} \\
b_{\gamma}
\end{array}\right)=A y(t)+B
$$


where $\boldsymbol{y}(\boldsymbol{t})$ is the target's coordinate in the y (superior-inferior) direction and the vectors $\boldsymbol{A}$ and $\boldsymbol{B}$ contain all scalars. Equation (3) relates all the components of equation (1) with the target's ycoordinate. This is advantageous because the gantry and $\mathrm{kV}$ imager rotate around the $\mathrm{y}$-axis. In fact, from the equation (2), we have:

$$
y(t \mid \theta)=\frac{S A D-(x(t) \cdot \cos \theta+z(t) \cdot \sin \theta)}{S I D} y_{p}(t)
$$

If we assume the quantity $(\boldsymbol{x}(\boldsymbol{t}) \cdot \boldsymbol{c o s} \boldsymbol{\theta}+\boldsymbol{z}(\boldsymbol{t}) \cdot \boldsymbol{s i n} \boldsymbol{\theta})$ in equation (4) to be much smaller than $\mathrm{SAD}$, which is realistic because SAD is normally $1000 \mathrm{~mm}$ in most clinical linac systems while the distance between the target position and the gantry rotation axis is typically small. This is because in reality, the radiation isocentre will either in or very close to the tumour, i.e. our target. In some cases, implanted markers are to be tracked instead of the tumour, which are usually implanted in the vicinity of the tumour. Then $y(t)$ can be approximated as:

$$
y(t)=\frac{S A D}{S I D} y_{p}(t)
$$

As equation (4-1) enables a relatively accurate estimation of $y(t)$, the scalar vectors $A$ and $B$ can hence be estimated using the least squares method, similar to methods described by Chung et al. (2016), Cho et al. (2012), Ruan et al. (2008), with some modifications: Firstly, from equation (3), $\left(\begin{array}{c}\widehat{\boldsymbol{T r}}_{\boldsymbol{x}} \\ \widehat{\boldsymbol{T r}}_{\boldsymbol{y}} \\ \widehat{\boldsymbol{T r}}_{\boldsymbol{z}} \\ \widehat{\boldsymbol{\alpha}} \\ \widehat{\boldsymbol{\beta}} \\ \widehat{\gamma}\end{array}\right)(\boldsymbol{t})$ can be estimated from $y(t)$. Consequently, we can compute the estimated coordinates $\left(\begin{array}{l}\widehat{\boldsymbol{x}} \\ \hat{\boldsymbol{y}} \\ \widehat{\boldsymbol{z}}\end{array}\right)$ using equation (1), which can then be used to compute the estimated projected coordinates $\left(\begin{array}{l}\widehat{\boldsymbol{x}}_{\boldsymbol{p}} \\ \widehat{\boldsymbol{y}}_{\boldsymbol{p}}\end{array}\right)$ by applying equation (2) to $\left(\begin{array}{l}\widehat{\boldsymbol{x}} \\ \widehat{\boldsymbol{y}} \\ \widehat{\boldsymbol{z}}\end{array}\right)$. The cost function of the Euclidean distance between $\left(\begin{array}{l}\widehat{x}_{\boldsymbol{p}} \\ \widehat{\boldsymbol{y}}_{\boldsymbol{p}}\end{array}\right)$ and the actual coordinates of the target $\left(\begin{array}{l}\boldsymbol{x}_{\boldsymbol{p}} \\ \boldsymbol{y}_{\boldsymbol{p}}\end{array}\right)$ can then be computed as:

$$
C=\left\|\sqrt{\left(x_{p}-\widehat{x}_{p}\right)^{2}+\left(y_{p}-\widehat{y}_{p}\right)^{2}}\right\|^{2}
$$

Finally, the vectors $\boldsymbol{A}$ and $\boldsymbol{B}$ can be estimated by minimizing the cost function $C$, given $\left(\begin{array}{l}x_{p} \\ y_{p}\end{array}\right)(t)$, in the least square sense. 
Since equation (4-1) is only an approximation of equation (4), we iteratively refine the solution, as shown the pseudo-codes in Figure 1.

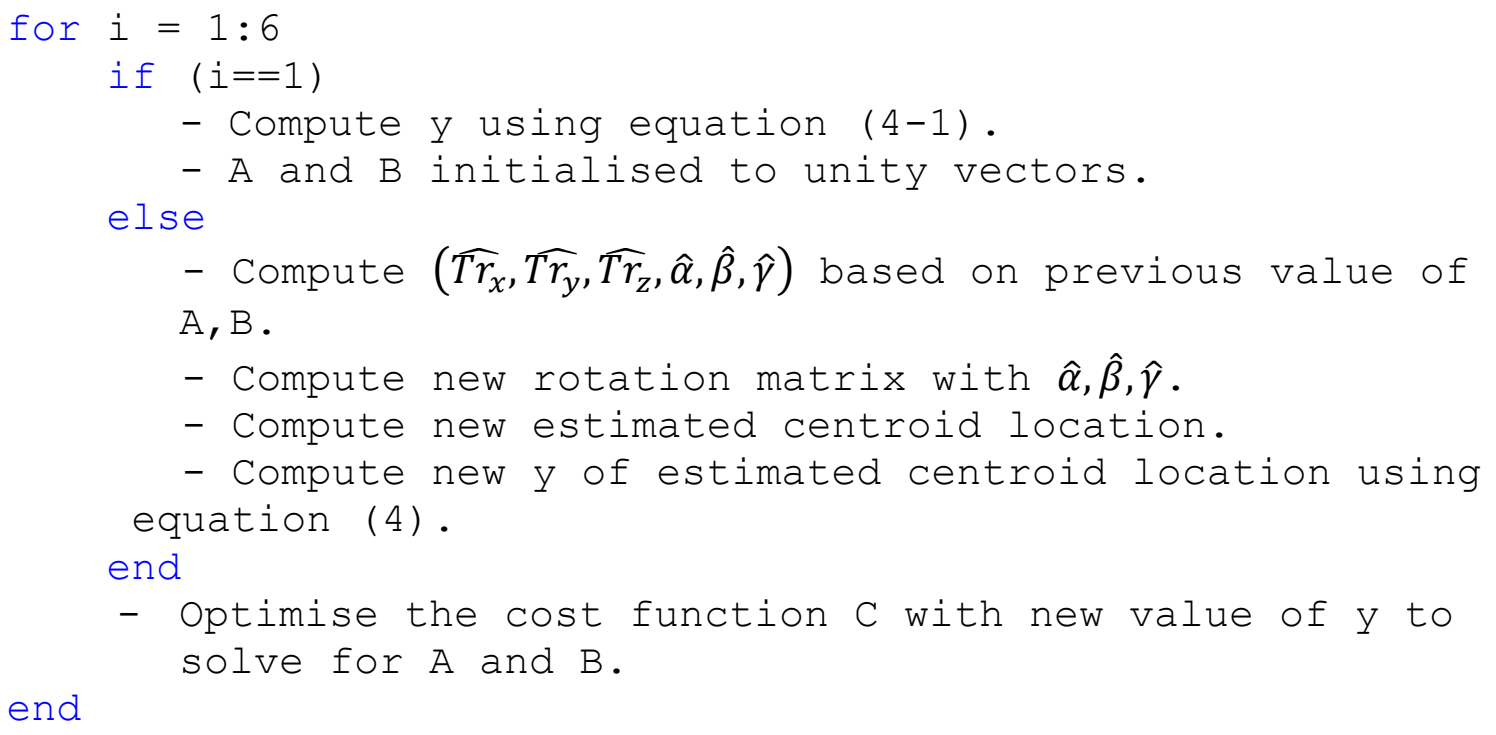

Figure 1. Pseudo-codes for refining vectors $A$ and $B$ estimations with $6 D-I D C$.

The flowchart in Figure 2 summarises in silico simulation and data processing to evaluate the accuracy of the proposed 6D-IDC algorithm.

\subsubsection{Ground-truth data descriptions}

A dataset of 29 patients with 3 fiducials implanted near the tumour in the liver for image guided radiotherapy, first described by Bertholet et al. (2016), was used in our in silico simulation. Each patient was treated with Stereotactic Body Radiation Therapy (SBRT), receiving treatment in 3-6 fractions. In each fraction, each patient's 1 to 3 cone beam CBCT scans $(11 \mathrm{fps}, 125 \mathrm{kV}, 80 \mathrm{~mA}, 13$ $\mathrm{ms}$ ) were acquired. The fiducials were segmented in each image. We furthermore rejected traces where the data were not continuous for at least 50 seconds. This is because the simulation aims to mimic real-time treatment to assess the algorithm's accuracy for realistic intrafraction conditions. If there were more than one segments of continuous data within one fraction, each continuous segment was used independently. Overall, the refined dataset contained 81 traces from 19 patients. The range of $6 \mathrm{DoF}$ motions in this dataset are shown in Figure 3. 
The ground truth 6 DoF motion data were computed in two steps. The $2 \mathrm{D} \rightarrow 3 \mathrm{D}$ estimation of each marker's position in each image frame was computed using the method of Poulsen et al. (2008b), which has been measured to have sub-mm accuracy (Keall et al., 2016, Poulsen et al., 2008b). From the imaging frames with all 3 markers successfully segmented, the 6DoF motions of the target were calculated using the ICP algorithm (Tehrani et al., 2013), which computed the 6DoF motions from individual 3D coordinates of the three markers. The accuracy of this method in estimating the rotation motions during radiotherapy were evaluated and quantified by Kim et al. (2016) and found to be accurate within $1^{\circ}$. The positions of the markers at the first frame of imaging in each fraction were used as the referenced positions for $6 \mathrm{DoF}$ calculation as it was the intrafraction $6 \mathrm{DoF}$ motion that were of interest.

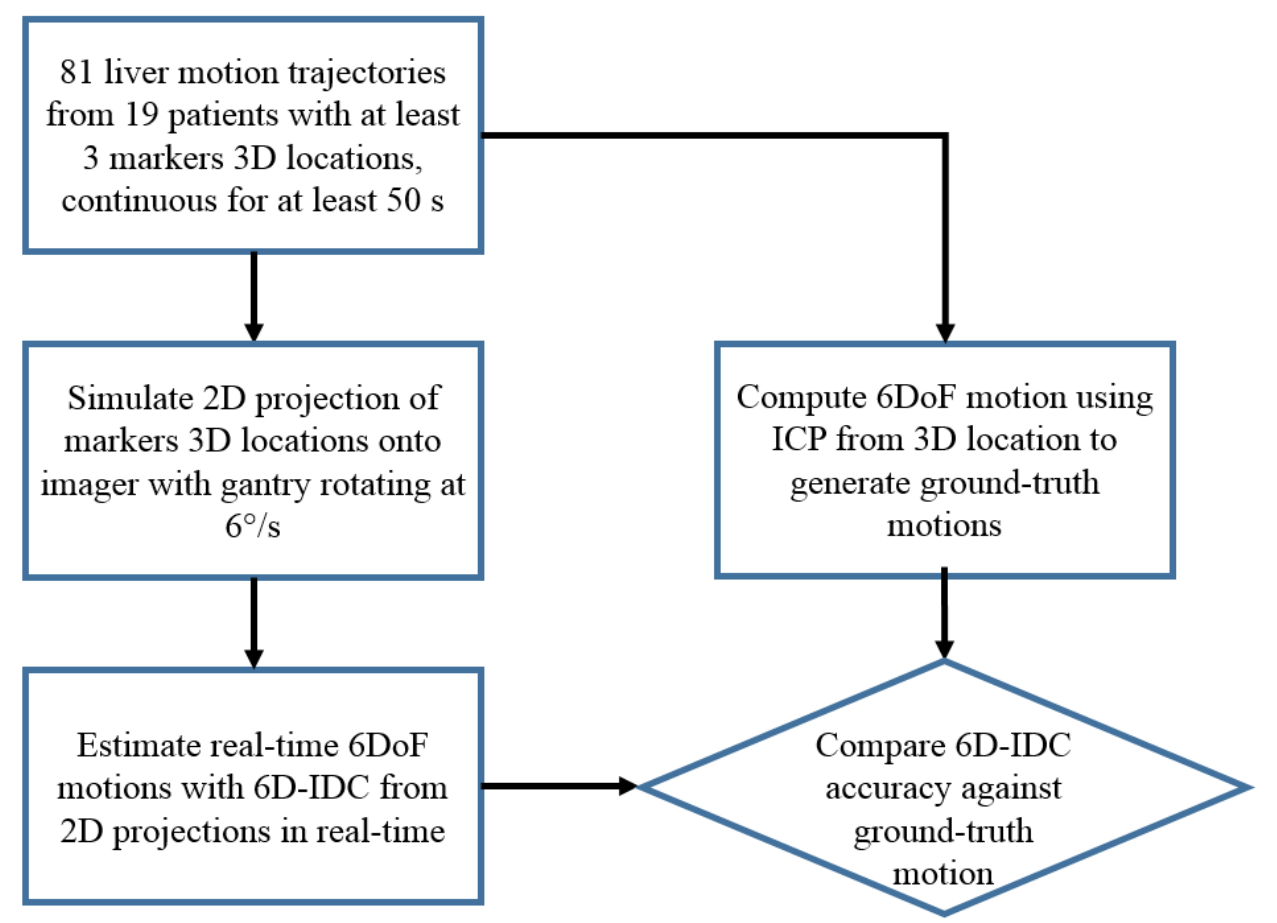

Figure 2. Flow chart of the in silico simulation for evaluating the accuracy of 6D-IDC. 

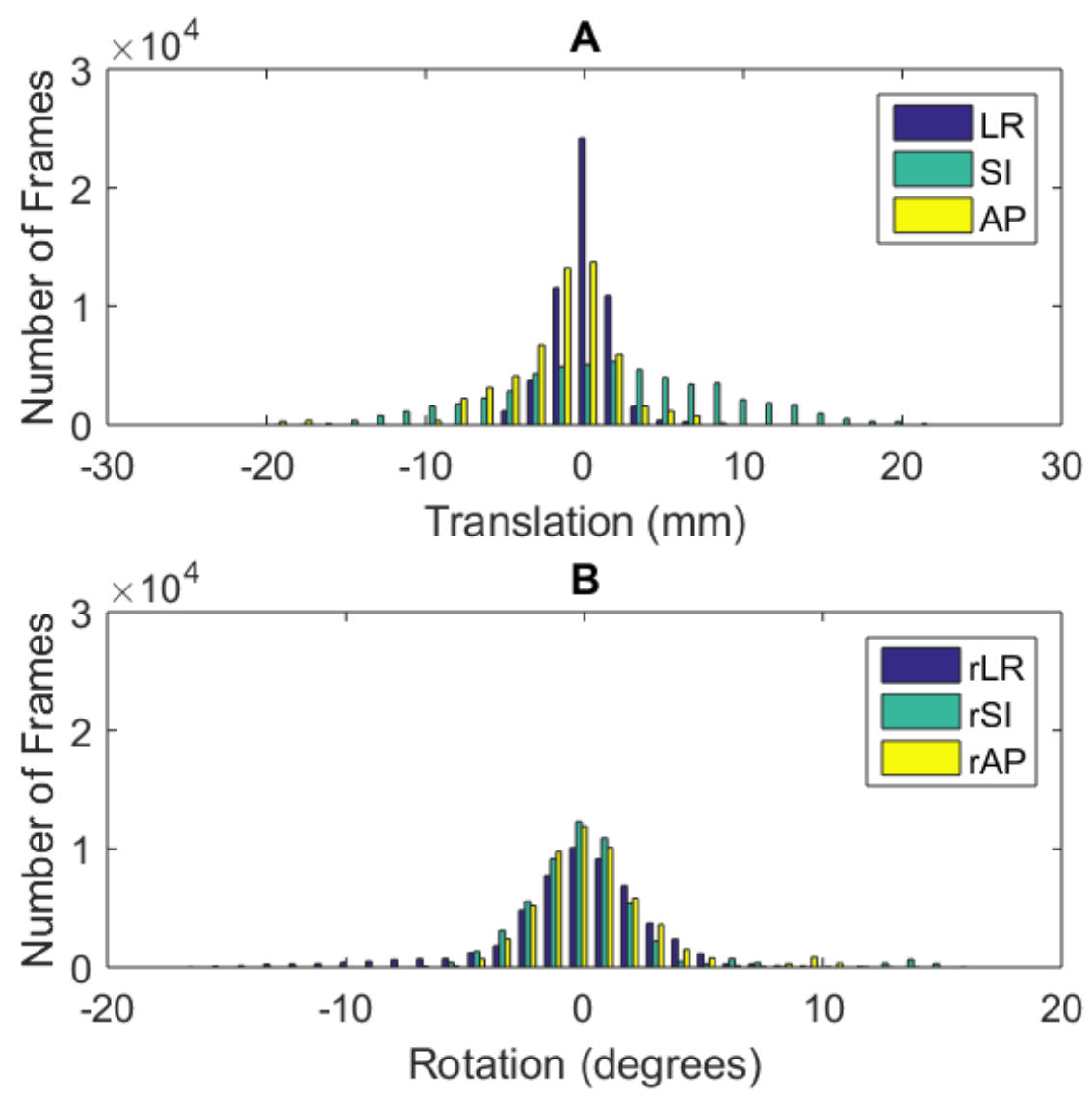

Figure 3. Histogram of six degrees of freedom motion in the ground-truth data, across 81 traces from 19 patients and 53736 frames. A: translational motion. B: rotational motion.

\subsubsection{VMAT simulation}

5 In order to test the accuracy of the 6D-IDC algorithm estimated 6DoF motion, for each trace in the ground-truth dataset, the ground-truth 3D positions of the markers were projected onto the imager using equation (2). The SAD and SID value were set at $1000 \mathrm{~mm}$ and $1800 \mathrm{~mm}$, respectively. For each simulation, the gantry started at $180^{\circ}$ and rotated counter-clockwise at $6 \%$ s to simulate a full rotation VMAT treatment.

The $6 \mathrm{D}-\mathrm{IDC}$ algorithm was then used to estimate $6 \mathrm{DoF}$ motion using only information from the projected positions of the markers on each image frame, as described in section 2.1. The first 6DIDC model was built after 200 imaging frames, equivalent to $110^{\circ}$ of gantry rotation. After the first model was updated, for each new frame, using all the data from the beginning of the treatment. However, when updating the model, only one iteration of optimisation was used, instead of 6 as described in the algorithm in Figure 1, and the least square optimisation was started at the last found solution for the vectors correlation vectors $A$ and $B$. For the first model, it was found that using 6 iteration allows the solution to converge for all the test trajectories with the difference in the sum of square error criterion set at 1e-6 mm. During the update phase, the least square solver used the solution from the last time point, this effectively gives it a "warm start". Thus, the six iterations were not necessary and one iteration was sufficient to have to solution converged. 


\subsubsection{Analysis of simulation results}

The error of the 6D-IDC algorithm was defined as the difference between 6DoF motions estimated with $6 \mathrm{D}-\mathrm{IDC}$ and the $6 \mathrm{DoF}$ ground-truth motion.

We analysed the factors affecting the accuracy of 6D-IDC, including:

5 1. Deformation: estimated by the change in the area of the triangle, that was formed by the 3 markers, in 3D in each frame, compared with the referenced area.

2. Absolute magnitude of motion in each DoF: the absolute value of 6DoF motions in each frame relative to planned marker position

3. Linear correlation between motion in each DoF and the motion of the SI direction: defined by the absolute value of the Pearson's linear correlation value $(\rho)$ computed between the motion in each DoF and the motion in SI for each tested trace.

The effect of each of the aforementioned factors to the accuracy was quantified by calculating the Pearson's correlation value $(\rho)$ between the absolute value of the error in each DoF and the tested parameter, except for the third factor, linear correlation value. The correlation between the maximum value of error in estimating 6DoF motion and the linear correlation value of each DoF was used instead because linear correlation value was a trace-specific value.

The 6D-IDC algorithm, in silico simulation and analyses of the results were implemented in Matlab (Mathworks, MA, USA). 


\section{Results}

For this section, the translational motion is denoted by its axis of motion, e.g, translation motion in LR is denoted as "LR". The rotational motion is denoted by an " $r$ " before its axis of rotation, e.g rotation motion around the SI axis is denoted as "rSI". This is simply for clarity in figures.

\subsection{Accuracy of $6 D-I D C$}

Figure 4 shows a comparison of 6DoF motion estimated using 6D-IDC and the ground truth motion used in the simulation. The means and standard deviations of the differences are summarised in Table 1 . The mean of error in the $6 \mathrm{DoF}$ are under $0.1 \mathrm{~mm}$ and $0.1^{\circ}$ across 81 motion traces from 19 patients. The standard deviation of error for 6D-IDC estimated motion are less than $1 \mathrm{~mm}$ for translational motion and less than $1.5^{\circ}$ for rotational motion. This result is a pooled analysis across 53736 imaging frames of the 81 liver motion traces from 19 patients. The boxplot of the overall error is shown in Figure 5.

Figure 6 shows the boxplot of the mean of error of 6D-IDC estimations compared with groundtruth 6 DoF motion for each of the 81 tested traces. From Figure 6, it can be observed than even though there are outliers of error up to $20^{\circ}$, as seen in Figure 5, in $95 \%$ of cases, the mean error of 6D-IDC estimated motion of each trace are within $1 \mathrm{~mm}$ and $1^{\circ}$. A typical example of one case where such large outliers are present, is shown in Figure 7. As depicted in Figure 7, some of the outliers were caused by sudden motion, such as coughing, which alter the linear relationship between the motion in SI and motions in the other DoFs. 
Table 1. Summary of error of 6DoF motion estimated with 6D-IDC.

\begin{tabular}{|c|c|c|c|}
\hline & $\begin{array}{l}\text { Mean } \\
\text { error }\end{array}$ & $\begin{array}{c}\text { Standard } \\
\text { deviation of error }\end{array}$ & $\begin{array}{c}{\left[5^{\text {th }} \text { to } 95^{\text {th }}\right]} \\
\text { percentile interval }\end{array}$ \\
\hline$L R(m m)$ & -0.03 & 0.32 & {$[-0.55-0.50]$} \\
\hline$S I(m m)$ & -0.01 & 0.13 & {$[-0.18-0.17]$} \\
\hline$A P(\mathrm{~mm})$ & 0.03 & 0.52 & {$[-0.64-0.73]$} \\
\hline rLR (degrees) & 0.07 & 1.18 & {$[-1.51-1.70]$} \\
\hline rSI (degrees) & 0.07 & 1.00 & {$[-1.50-1.70]$} \\
\hline rAP (degrees) & 0.06 & 1.32 & {$[-1.53-1.68]$} \\
\hline
\end{tabular}



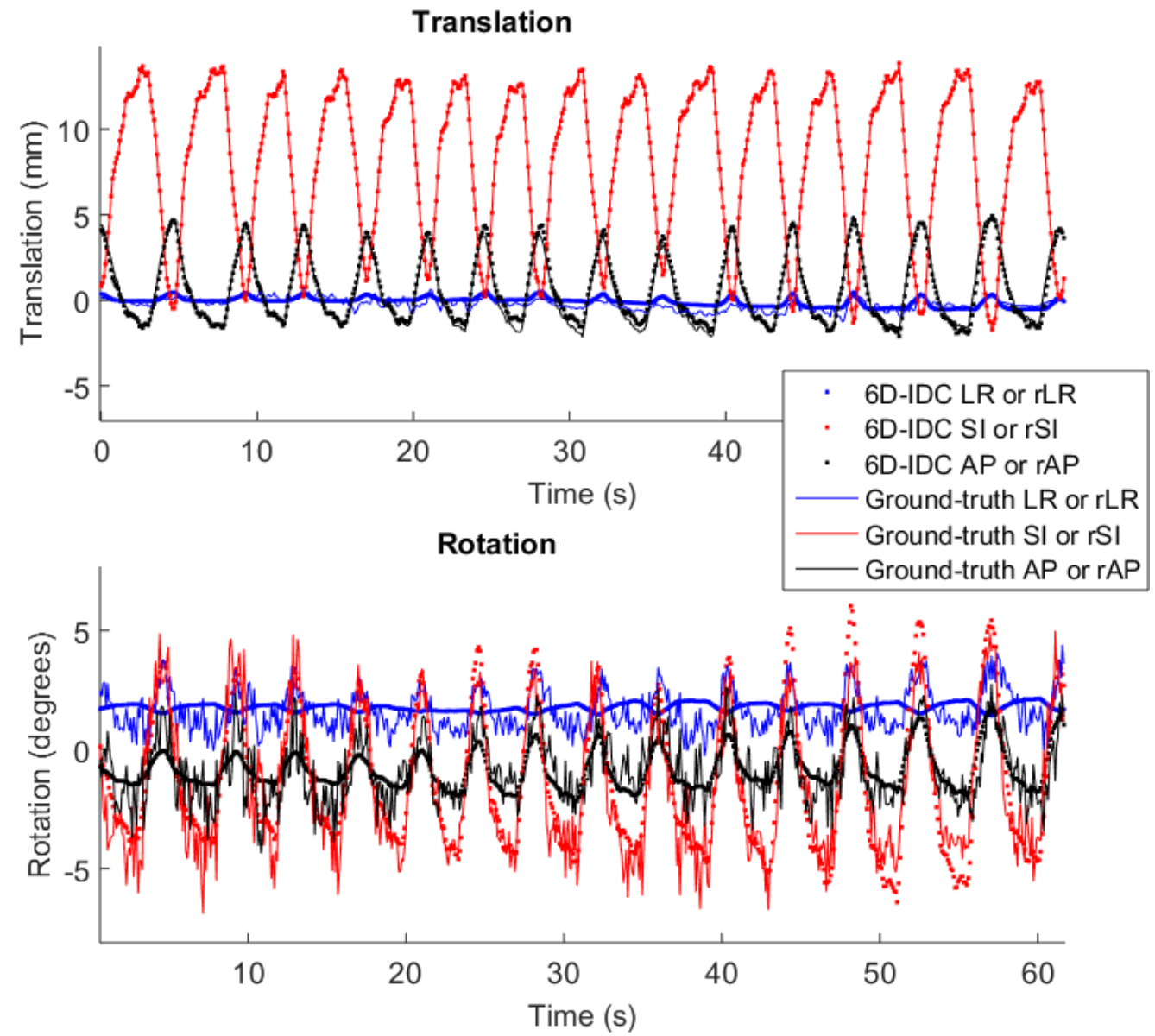

Figure 4. Six degree of freedom target motion successfully estimated with 6D-IDC compared to the ground truth. The data is from patient 26 , fraction 3. 


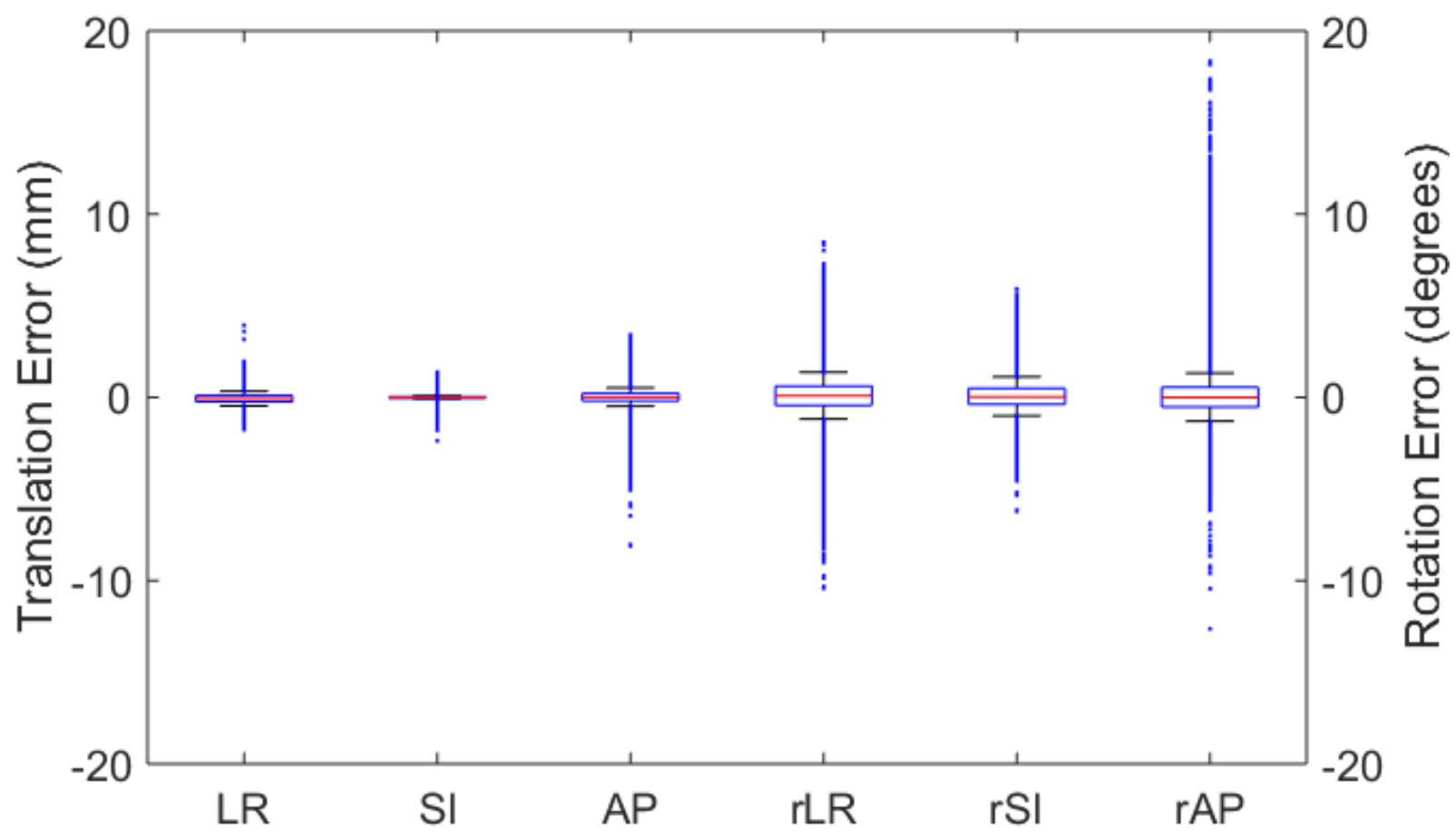

Figure 5. Boxplot showing the distributions of error in 6DoF between 6D-IDC estimation and ground-truth across 81 liver tumour traces from 19 patient with 53736 image frames.

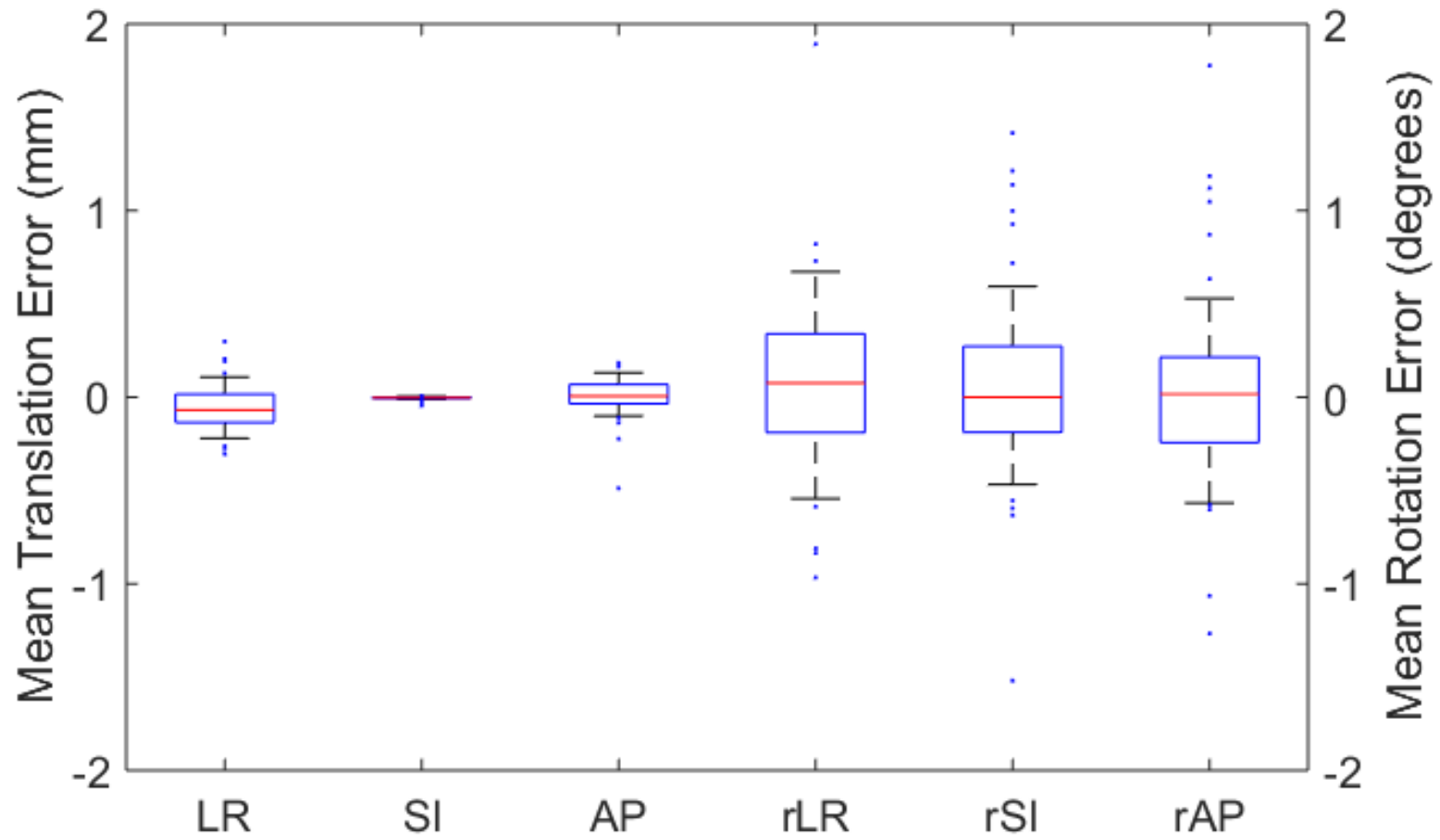

$5 \quad$ Figure 6. Boxplot showing the distributions of the mean of error in each segment in 6DoF between 6D-IDC estimation and ground-truth across 81 liver tumour traces from 19 patients. 


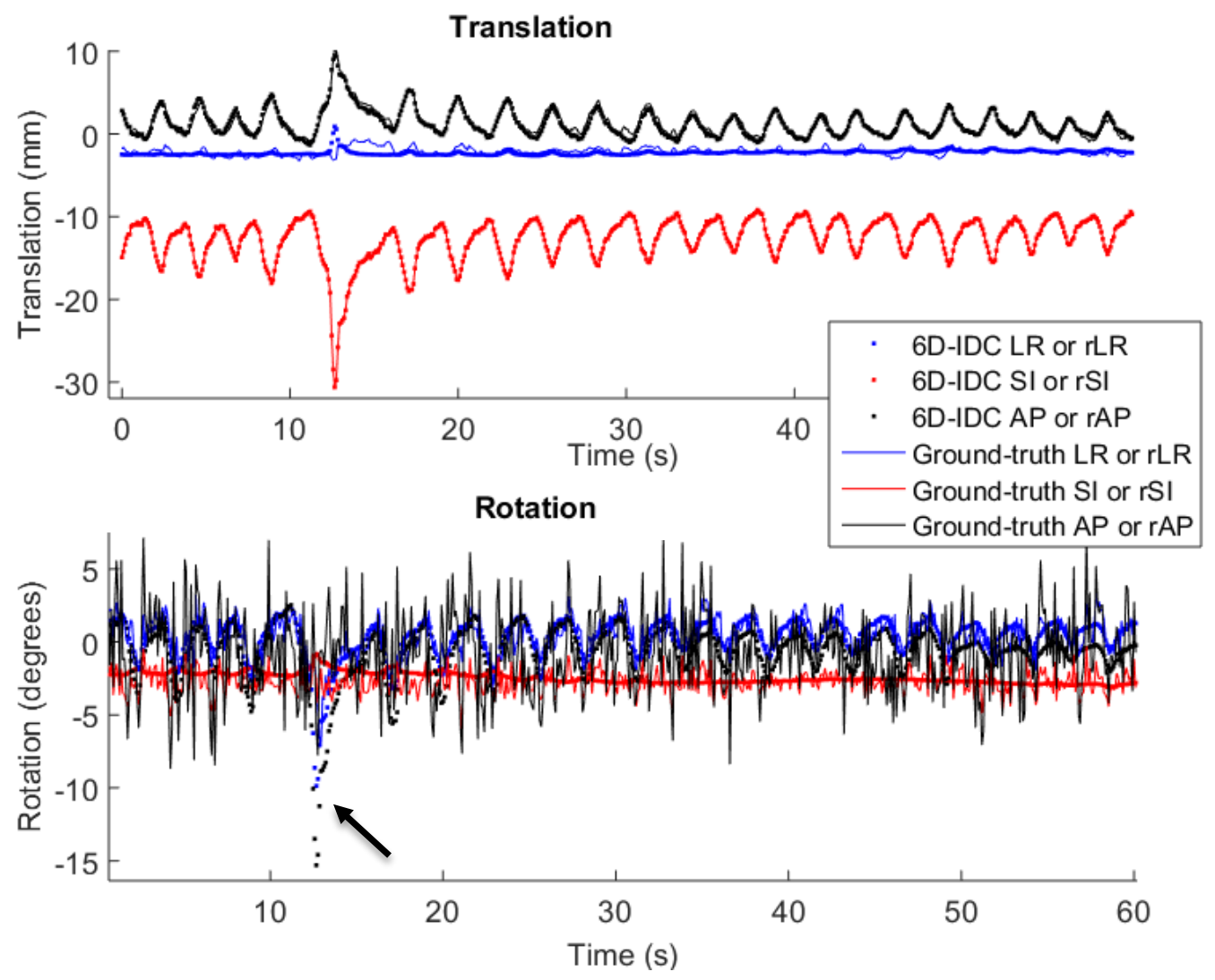

Figure 7. An example where 6D-IDC struggled in estimating motion due to sudden change in correlation (arrow). The data from patient 14, fraction 1.

\subsection{Factors affecting 6D-IDC accuracy}

5 Figures 8 and 9 are the scatter plots of the error between 6D-IDC estimated motions in each DoF as a function of the magnitude of deformation, assessed by the variation in the area of the triangle subtended by the markers (Figure 8) and the absolute value of motion (Figure 9).

The magnitude of the deformation seen in the ground-truth dataset had little effect on the accuracy of the 6D-IDC algorithm (Figure 8). However, from the scatter plots in figure 8, the relationship between the magnitude of error and the change in area in each frame was weak in all six DoFs. The computed Pearson correlation coefficients shows that the error in the translational SI direction has the highest correlation with the change in area $(\rho=0.41)$, followed by the error in the translational AP direction $(\rho=0.28)$ and the error in the rotation around the LR axis $(\rho=$ 0.22 ). 

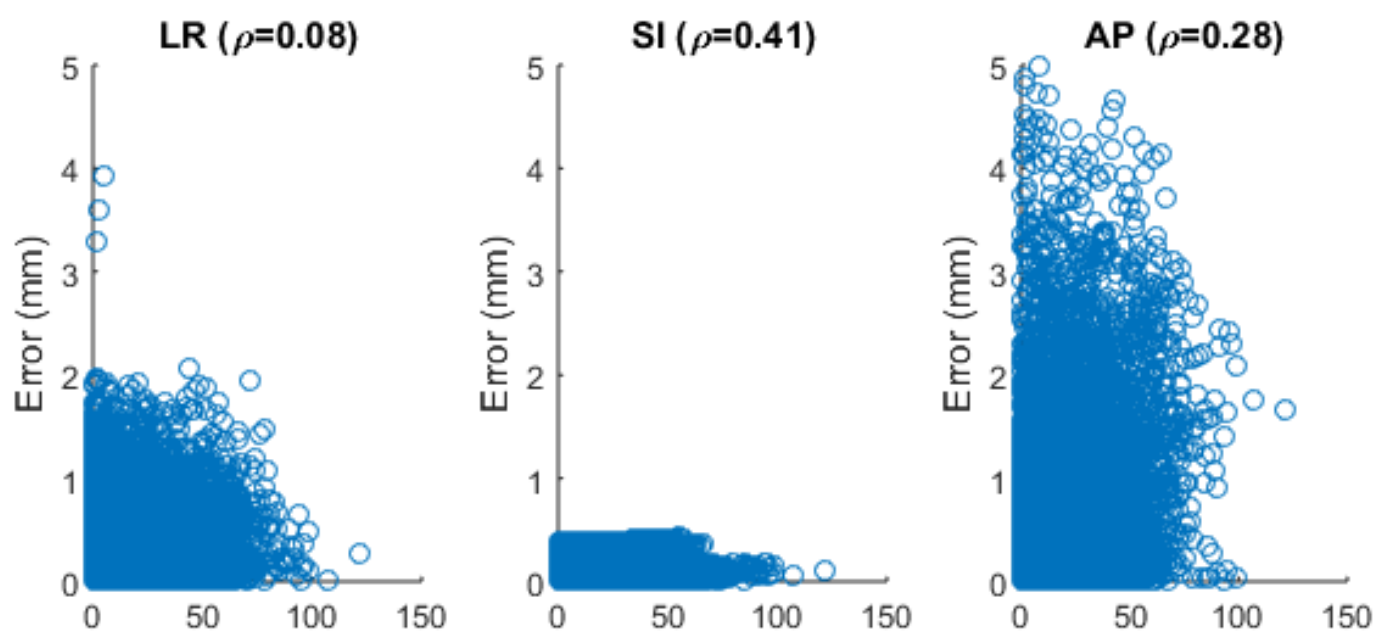

Change in area $\left(\mathrm{mm}^{2}\right)$

Change in area $\left(\mathrm{mm}^{2}\right)$

Change in area $\left(\mathrm{mm}^{2}\right)$
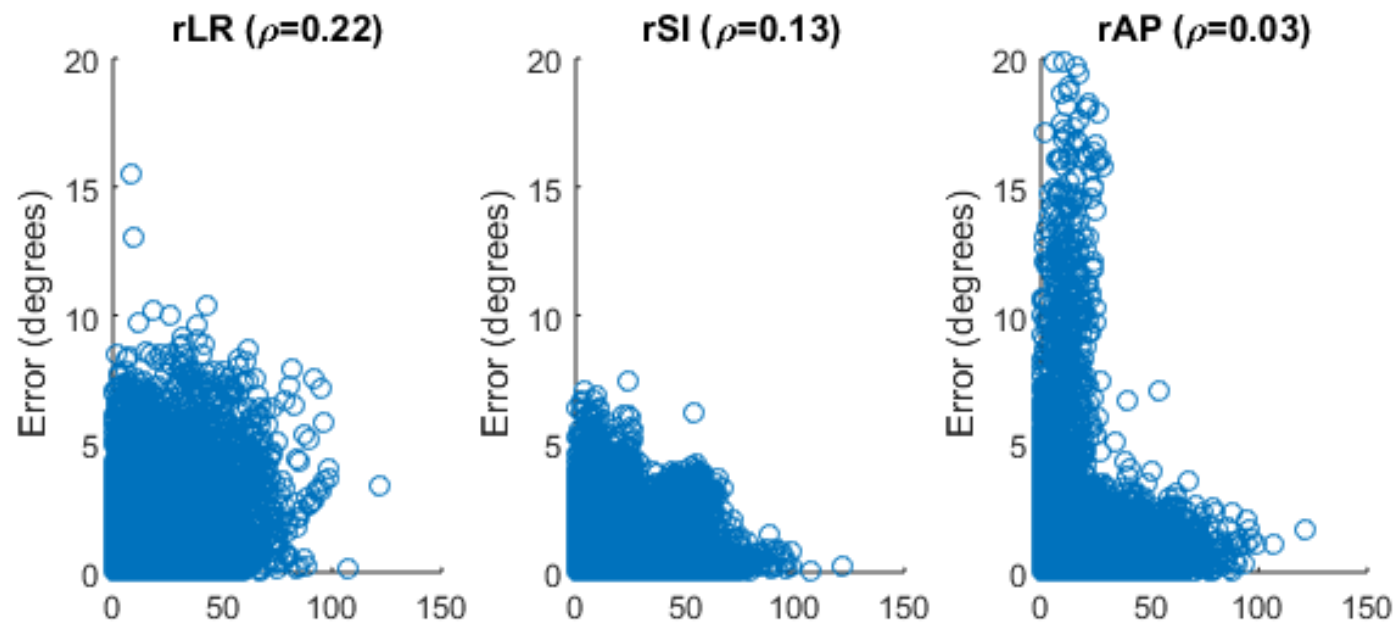

Change in area $\left(\mathrm{mm}^{2}\right) \quad$ Change in area $\left(\mathrm{mm}^{2}\right)$

Change in area $\left(\mathrm{mm}^{2}\right)$

Figure 8. Scatter plot showing the relationship between the magnitude of errors and the magnitude of the change in the area made by the three fiducials. The $\rho$ value indicates the Pearson correlation coefficient between each value pair.

5 The absolute magnitude of ground-truth motion, which includes systematic offsets, does not have any effect on the magnitude of 6D-IDC error in estimating translational motion; all the Pearson's correlation $\rho$ values for the translation motion are less than 0.1 (Figure 9). The magnitude of the rotational motions are weakly correlated with the magnitude of the error (Figure 9) with rSI and rAP having $\rho$ values less than 0.3 and rLR having a $\rho$ value of 0.42 . 

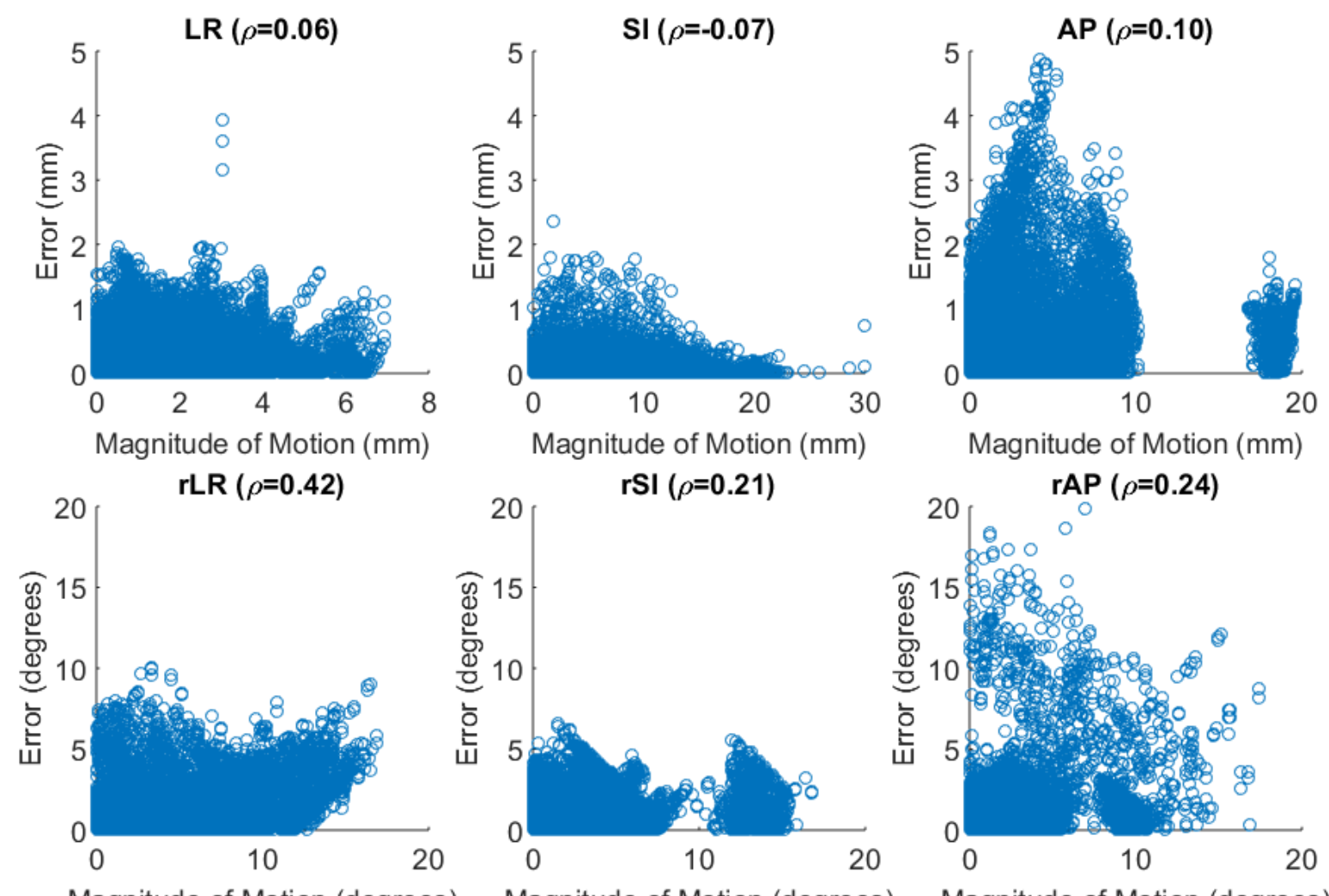

Magnitude of Motion (degrees)

Magnitude of Motion (degrees)

Magnitude of Motion (degrees)

Figure 9. Scatter plots showing the relationship between the magnitude of errors and the magnitude of absolute motion of the target. The $\rho$ value indicates the Pearson correlation coefficient between each value pair.

Figure 10 shows scatter plots of the maximum of error and the linear correlation between each DoF motion and the translational SI motion for all tested traces. A strong correlation is found in the AP translation motion and the rotation around the LR axis (rLR), with Pearson's correlation $\rho$ values of -0.6 for AP and -0.5 for rLR. A negative Pearson's correlation indicates a negatively correlated relationship. However, in all other DoF motions, no correlation or very weak correlation can be observed. From Figure 10, it can also be observed that most of the outliers occurred with weak correlation with SI $(<0.2)$, especially in translation motions in AP and rLR and rAP rotation motion. 

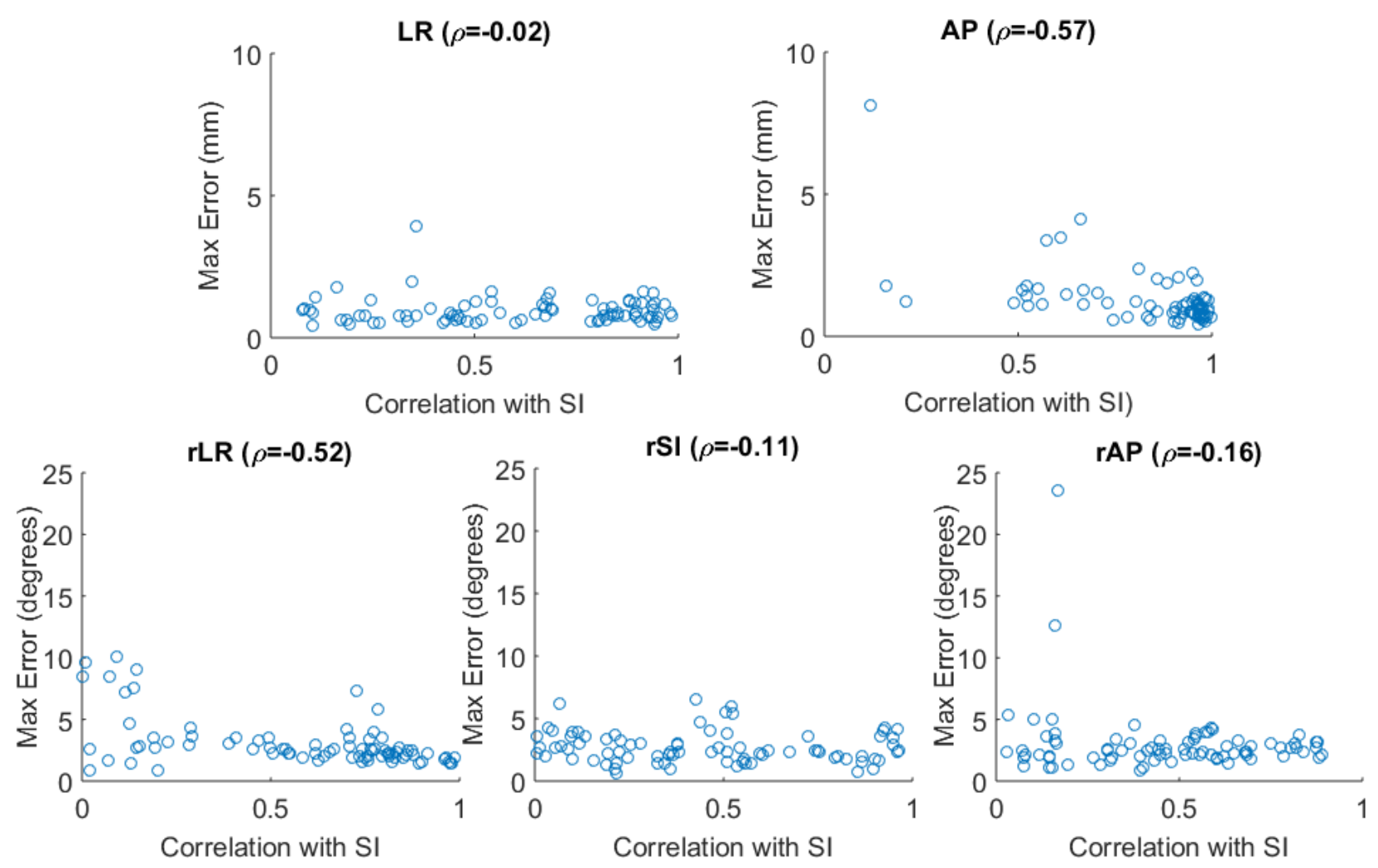

Figure 10. Scatter plots showing the relationship between the maximum error of 6D-IDC estimations in each degree of freedom and the absolute linear correlation value of the motion in that degree of freedom and the motion in the SI direction for each trace. The $\rho$ value indicates the Pearson correlation coefficient between each value pair. 


\section{Discussion}

This paper describes a method to directly estimate real-time 6DoF target motion from segmented marker positions on a 2D imager that is orthogonally mounted on the gantry of a standard linac. Previous efforts to calculate $6 \mathrm{DoF}$ motion from the target's 2D positions were done in two steps: (i) estimating 3D position of the target; and (ii) calculating the $6 \mathrm{DoF}$ motion by iteratively minimising the sum of squared differences in estimated and measured 3D positions of the target (Tehrani et al., 2013, Huang et al., 2015, Bertholet et al., 2016). The presented method utilises the interdimensional correlation in the translation in SI direction with other 5 degrees of freedom motions as an a priori, which was found to exist for liver and lung traces in previous studies (Huang et al., 2015, Bertholet et al., 2016). This new method, named 6D-IDC, has been evaluated in a series of in silico simulations with liver SBRT patients' $6 \mathrm{DoF}$ trajectories. Compared with the ground-truth $6 \mathrm{DoF}$ motion, assumed as the current standard for estimating 6DoF motions, the 6D-IDC algorithm performed equivalently well, with sub-mm and sub-degree accuracy on the tested dataset. The accuracy (mean) and precision (standard deviation) of the 6D-IDC method in estimating translation motions of the tested dataset were sub-mm, which is comparable to previously reported 2D-3D accuracy of probability-based methods in similar simulations with thoracic/abdominal tumour trajectories (Li et al., 2011, Poulsen et al., 2008a).

The presented 6D-IDC algorithm employs solving the correlation matrix in a least square sense. This formalism of solving 6DoF motion from the target's projection on an imager is a scalable solution. In our simulation, three points, i.e. markers, were used. Three is the lowest number of points to describe the target that allows the algorithm to uniquely determine the rotation and translation of the object. The algorithm is capable of solving for the $6 \mathrm{DoF}$ motion of a target comprised of a larger number of points, such as situations with four or more markers, or the segmented tumour on a projection image.

The perspective-n-point problem in computer vision can be used to solve for the $6 \mathrm{DoF}$ information of an object, provided three or more points are available on an image taken by a calibrated camera ( $\mathrm{kV}$ imaging system in this case) and the distance between these points are known. Haralick et al. (1994) describes a range of algorithms to solve for the 3D position of each points of the object in the case where only three points are available, from which the absolute orientation of the object can be determined. Thus, the perspective-n-point problem is in fact similar to the problem of determining $6 \mathrm{DoF}$ tumour motions of radiotherapy. Similar to the existing method (Tehrani et al., 2013) to determine tumour translation and rotation from fluoroscopic 2D images, the perspective-n-point solves the translation and rotation in two steps. The $3 \mathrm{D}$ coordinates of each point must be solved before the absolute transformation matrix can be determined (Haralick et al., 1994). The advantage of our solution to solve for tumour motion during treatment is in the use of equation (3), which linearly models the correlation between motion in the SI direction and the other 5 degrees of freedom motions. Utilising equation 3, our algorithm is able to compute the rotation and translation of the target directly, without the need to solve for the 3D coordinates of each point separately.

The reported results were from an online scenario where the 6D-IDC algorithm estimated 6DoF motion on each new in-coming image, after a learning arc. The accuracy of the algorithm is likely 
to be better in a retrospective scenario. However, we were more interested in its performance in real-time as the main application for such an algorithm is intrafraction monitoring and as a positioning system for a tracking system. A learning arc of $110^{\circ}$ was used in our simulation, which is less than the amount of learning arc $\left(120^{\circ}\right)$ used in clinical application of the probability-based method by Poulsen et al. (2008a) (Keall et al., 2016). Additionally, Chung et al. (2016) described a 3D IDC-based method and showed that a learning arc of at least $90^{\circ}$ reduced the errors in estimating motions at the start of the trajectory. Similarly, we found that around $110^{\circ}$ of learning arc provided a stable start for the 6D-IDC algorithm.

As shown in figures 4 and 7, the rotation motions calculated with 6D-IDC tend to be smoother and free of spikes, compared with rotation motions calculated with the ICP algorithm. This is because the ICP algorithm is sensitive to noise in the $3 \mathrm{D}$ positions of the object. That is, if one of the three markers moves due to the noise in the system, the ICP algorithm interpreted such movements as true motions and attempted to over-fit into its model. Meanwhile, the presented 6DIDC algorithm attempted to find the best rotation and translation correlation parameters to minimise the error between the estimated position of the object and its projection on the imager. Effectively, performing the least square estimation on at least 200 points of data filtered the signal so as to mitigate noise in the estimated signal as well.

We investigated a number of factors that could influence the accuracy of the presented 6D-IDC algorithm, including deformation, quantified by the change in area of the triangle formed by the 3 markers in 3D, the magnitude of motion in $6 \mathrm{DoF}$ and the correlation between each DoF and the motion in SI. Among these three factors, it was found that the algorithm was robust against both deformation and both large rotation and translation motions, with low or very low Pearson's correlation value between the magnitude of error and any of the aforementioned values. However, the 6D-IDC algorithm was sensitive to the correlation value between each DoF and the motion in SI, especially in the AP and rLR direction. The outliers in the estimation errors of each DoF also occur more frequently at low correlation, as shown in Figure 10. If the algorithm is to be implemented clinically, the correlation value can be used to assess the degree of its reliability in estimating 6DoF motion in each patient. In patients whose motions have low value of correlation, e.g correlation with magnitude of less than 0.2 , probability methods such as those described by (Poulsen et al., 2008b, Li et al., 2011) could be more accurate than the 6D-IDC method.

\section{Limitations and future work}

One limitation of this work is the inherent uncertainty of the ground-truth dataset. From the previous works (Bertholet et al., 2016, Keall et al., 2016, Kim et al., 2017, Poulsen et al., 2008a, Poulsen et al., 2008b), it has been shown that the uncertainty of the ground-truth data set is within $1 \mathrm{~mm}$ and $1^{\circ}$. Specifically, Kim et al. (2017) showed experimentally on a phantom that estimation of $6 \mathrm{DoF}$ motions for prostate and lung tumour traces using the combination of the probability method and the ICP method has an accuracy of $1 \mathrm{~mm}$ and $1^{\circ}$ as compared with $\mathrm{kV} / \mathrm{MV}$ triangulation. Furthermore, the ground-truth data rejected 3D points that had an uncertainty of more than $1 \mathrm{~mm}$, as described in (Bertholet et al., 2016). Although the uncertainty of the ground-truth dataset is low, it does affect the presented results. In the worst-case scenario, the reported error is an additive combination of the actual error inherent to our method and the inherent error within the groundtruth dataset. Data from a more independent source such as the electromagnetic system Calypso 
(Varian Medical System, Palo Alto, CA, USA) may provide a more reliable benchmarking data than the dataset used in this paper, e.g. in clinical trials such as (Poulsen et al., 2016, Booth et al., 2016). However, these clinical trials have not been completed and the data is not yet available.

In this paper, the 6D-IDC method was evaluated using in silico simulations for VMAT 5 treatments. Due to the short duration of the continuous trajectories available in our dataset where all of the tested trajectories were less than 65 seconds in length, IMRT simulations were not carried out. As more data of intrafraction tumour motions of the thoracic and abdominal tumours with at least 3 points becomes available from the ongoing clinical trials, the algorithm will also be evaluated for IMRT treatments. The immediate next step is to further evaluate the accuracy of the algorithm in phantom experiments, paving the way to develop it as a clinical tool for real-time tumour motion management during treatment. Such a development would require the algorithm to be implemented together with a marker or markerless tumour segmentation algorithm.

\section{Conclusion}

This paper describes, to the best of our knowledge, the first method that is capable of directly estimating real-time $6 \mathrm{DoF}$ target motion from the target's positions on a 2D imager that is mounted on the gantry of a standard linear accelerator.. The accuracy of the algorithm was numerically evaluated using 81 liver tumour motion traces from 19 patients and found to be within sub-mm and sub-degree, with precision sub-mm and sub-1.5 degrees. Requiring only images from a single rotating $\mathrm{kV}$ imaging system that is widely available in all modern linear accelerators, the described method can thus be used to estimate the real-time tumour $6 \mathrm{DoF}$ motion for real-time treatment adaptations.

\section{Acknowledgments}

This work is funded by a Cancer Australia grant (Priority-driven Collaborative Cancer Research Scheme). Author P Keall is funded by an Australian NHMRC Senior Professorial Research Fellowship. We especially thank the Department of Radiation Oncology at Aarhus University Hospital (Denmark) for their generosity in sharing anonymised tumour trajectories which was used in this paper for evaluating our novel algorithm. 


\section{References}

Amro, H., Hamstra, D. A., Mcshan, D. L., Sandler, H., Vineberg, K., Hadley, S. \& Litzenberg, D. 2013. The Dosimetric Impact of Prostate Rotations During Electromagnetically Guided External-Beam Radiation Therapy. Int J Radiat Oncol Biol Phys, 85, 230-236.

Aubry, J.-F., Beaulieu, L., Girouard, L.-M., Aubin, S., Tremblay, D., Laverdière, J. \& Vigneault, E. 2003. Measurements of intrafraction motion and interfraction and intrafraction rotation of prostate by three-dimensional analysis of daily portal imaging with radiopaque markers. Int J Radiat Oncol Biol Phys, 60, 30-39.

Ballhausen, H., Li, M., Hegemann, N.-S., Ganswindt, U. \& Belka, C. 2015. Intra-fraction motion of the prostate is a random walk. Phys Med Biol, 60.

Becker, N., Smith, W. L., Quirk, S. \& Kay, I. 2010. Using cone-beam CT projection images to estimate the average and complete trajectory of a fiducial marker moving with respiration. Phys Med Biol, 55, 7439-7452.

Bertholet, J., Worm, E. S., Fledelius, W., Høyer, M. \& Poulsen, P. R. 2016. Time-Resolved Intrafraction Target Translations and Rotations During Stereotactic Liver Radiation Therapy: Implications for Marker-based Localization Accuracy. Int J Radiat Oncol Biol Phys, 95, 802-809.

Booth, J. T., Caillet, V., Hardcastle, N., O’brien, R., Szymura, K., Crasta, C., Harris, B., Haddad, C., Eade, T. \& Keall, P. J. 2016. The first patient treatment of electromagnetic-guided real time adaptive radiotherapy using MLC tracking for lung SABR. Radiother Oncol, 121, 1925.

Castellanos, E., Ericsson, M. H., Sorcini, B., Green, U., Nilsson, S. \& Lennernäs, B. 2012. RayPilot - electromagnetic real-time positioning in radiotherapy of prostate cancer - initial clinical results. Radiother Oncol, 103, S433.

Cho, B., Poulsen, P. R., Ruan, D., Sawant, A. \& Keall, P. J. 2012. Experimental investigation of a general real-time 3D target localization method using sequential $\mathrm{kV}$ imaging combined with respiratory monitoring. Phys Med Biol, 57, 7395-7407.

Chung, H., Poulsen, P. R., Keall, P. J., Cho, S. \& Cho, B. 2016. Reconstruction of implanted marker trajectories from cone-beam $\mathrm{CT}$ projection images using interdimensional correlation modeling. Med Phys, 43, 4643-4654.

Fallone, B. G., Murray, B., Rathee, S., Stanescu, T., Steciw, S., Vidakovic, S., Blosser, E. \& Tymofichuk, D. 2009. First MR images obtained during megavoltage photon irradiation from a prototype integrated linac-MR system. Med Phys, 36, 2084-2088.

Haralick, R. M., Lee, C.-N., Ottenberg, K. \& Nolle, M. 1994. Review and analysis of solutions of the three point perspective pose estimation problem. International Journal of Computer Vsion, 13, 331--356.

Huang, C.-Y., Tehrani, J. N., Ng, J. A., Booth, J. T. \& Keall, P. J. 2015. Six Degrees-of-Freedom Prostate and Lung Tumor Motion Measurements Using Kilovoltage Intrafraction Monitoring. Int J Radiat Oncol Biol Phys, 91, 368-375.

Keall, P. J., Ng, J. A., Juneja, P., O’brien, R. T., Huang, C.-Y., Colvill, E., Caillet, V., Simpson, E., Poulsen, P. R., Kneebone, A., Eade, T. \& Booth, J. T. 2016. Real-Time 3D Image Guidance Using a Standard LINAC: Measured Motion, Accuracy, and Precision of the First Prospective Clinical Trial of Kilovoltage Intrafraction Monitoring Guided Gating for Prostate Cancer Radiation Therapy. Int J Radiat Oncol Biol Phys, 94, 1015-1021.

Kim, J.-H., Nguyen, D. T., Huang, C.-Y., O’brien, R., Caillet, V., Poulsen, P. R., Booth, J. T. \& Keall, P. Quantifying the Accuracy and Precision of Six Degree-Of-Freedom Motion Estimation for Use in Real-Time Tumor Motion Monitoring During Radiotherapy. The 58th Annual Meeting of the American Association of Physicists in Medicine, 2016. Medical Physics, 3858-3859.

50 Kim, J., Nguyen, D., Huang, C., Fuangrod, T., Caillet, V., O'brien, R., Poulsen, P., Booth, J. \& Keall, P. 2017. Quantifying the accuracy and precision of a novel real-time 6 degree-of- 
freedom kilovoltage intrafraction monitoring (KIM) target tracking system. Physics in medicine and biology, 62, 5744-5759.

King, C. R., Brooks, J. D., Gill, H., Pawlicki, T., Cotrutz, C. \& Presti, J. C. 2009. Stereotactic body radiotherapy for localized prostate cancer: Interim results of a prospective phase II clinical trial. Int J Radiat Oncol Biol Phys, 73, 1043-1048.

Kitamura, K., Shirato, H., Shimizu, S., Shinohara, N., Harabayashi, T., Shimizu, T., Kodama, Y., Endo, H., Onimaru, R., Nishioka, S., Aoyama, H., Tsuchiya, K. \& Miyasaka, K. 2002. Registration accuracy and possible migration of internal fiducial gold marker implanted in prostate and liver treated with real-time tumor-tracking radiation therapy (RTRT). Int $J$ Radiat Oncol Biol Phys, 62, 275 - 281.

Kupelian, P., Willoughby, T., Mahadevan, A., Djemil, T., Weinstein, G., Jani, S., Enke, C., Solberg, T., Flores, N., Liu, D., Beyer, D. \& Levine, L. 2007. Multi-institutional clinical experience with the Calypso System in localization and continuous, real-time monitoring of the prostate gland during external radiotherapy. Int J Radiat Oncol Biol Phys, 67, 1088-1098.

Li, R., Fahimian, B. P. \& Xing, L. 2011. A Bayesian approach to real-time 3D tumor localization via monoscopic x-ray imaging during treatment delivery. Med Phys, 38, 4205-4214.

Nguyen, D. T., Kim, J.-H., O’brien, R. T., Huang, C.-Y., Booth, J. T., Greer, P., Legge, K., Poulsen, P. R., Martin, J. \& Keall, P. J. 2017. The First Clinical Implementation of a Real-Time Six Degree of Freedom Tracking System During Radiation Therapy. Radiother Oncol, [In Press].

Park, J. C., Park, S. H., Kim, J. H., Yoon, S. M., Song, S. Y., Liu, Z., Song, B., Kauweloa, K., Webster, M. J., Sandhu, A., Mell, L. K., Jiang, S. B., Mundt, A. J. \& Song, W. Y. 2012. Liver motion during cone beam computed tomography guided stereotactic body radiation therapy. Med Phys, 39, 6431-6442.

Poulsen, P. R., Cho, B. \& Keall, P. J. 2008a. A METHOD TO ESTIMATE MEAN POSITION, MOTION MAGNITUDE, MOTION CORRELATION, AND TRAJECTORY OF A TUMOR FROM CONE-BEAM CT PROJECTIONS FOR IMAGE-GUIDED RADIOTHERAPY. Int J Radiat Oncol Biol Phys, 72, 1687-1596.

Poulsen, P. R., Cho, B., Langen, K., Kupelian, P. \& Keall, P. J. 2008b. Three-dimensional prostate position estimation with a single $\mathrm{x}$-ray imager utilizing the spatial probability density. Phys Med Biol, 53, 4331-4353.

Poulsen, P. R., Worm, E. S., Hansen, R., Larsen, L. P., Grau, C. \& Høyer, M. 2016. Respiratory gating based on internal electromagnetic motion monitoring during stereotactic liver radiation therapy: First Results. Acta Oncologica, 54, 1445-1452.

Raaymakers, B. W., Lagendijk, J. J. W., Overweg, J., Kok, J. G. M., Raaijmakers, A. J. E., Kerkhof, E. M., Put, R. W. V. D., Meijsing, I., Crijns, S. P. M. \& Benedosso, F. 2009. Integrating a 1.5 T MRI scanner with a 6 MV accelerator: proof of concept. Phys Med Biol, 54, N229.

Rijkhorst, E.-J., Lakeman, A., Nijkamp, J., Bois, J. D., Herk, M. V., Lebesque, J. V. \& Sonke, J.-J. 2009. Strategies for Online Organ Motion Correction for Intensity-Modulated Radiotherapy of Prostate Cancer: Prostate, Rectum, and Bladder Dose Effects. Int J Radiat Oncol Biol Phys, 75, 1254-1260.

Ruan, D., Fessler, J. A., Balter, J. M., Berbeco, R. I., Nishioka, S. \& Shirato, H. 2008. Inference of hysteretic respiratory tumor motion from external surrogates: A state augmentation approach. Phys Med Biol, 53, 2923-2936.

Sazawa, A., Shinohara, N., Harabayashi, T., Abe, T., Shirato, H. \& Nonomura, K. 2009. Alternative approach in the treatment of adrenal metastasis with a real-time tracking radiotherapy in patients with hormone refractory prostate cancer. Int J Urol., 16, 410-412.

Shimizu, S., Shirato, H., Kitamura, K., Shinohara, N., Harabayashi, T., Tsukamoto, T., Koyanagi, T. \& Miyasaka, K. 2000. Use of an implanted marker and real-time tracking of the marker for the positioning of prostate and bladder cancers. Int J Radiat Oncol Biol Phys, 48, 15911597. 
Shirato, H., Harada, T., Harabayashi, T., Hida, K., Endo, H., Kitamura, K., Onimaru, R., Yamazaki, K., Kurauchi, N., Shimizu, T., Shinohara, N., Matsushita, M., Dosaka-Akita, H. \& Miyasaka, K. 2003. Feasibility of insertion/implantation of 2.0-mm-diameter gold internal fiducial markers for precise setup and real-time tumor tracking in radiotherapy. Int $J$ Radiat Oncol Biol Phys, 56, 240-247.

Shirato, H., Shimizu, S., Kunieda, T., Kitamura, K., Van Herk, M., Kagei, K., Nishioka, T., Hashimoto, S., Fujita, K., Aoyama, H., Tsuchiya, K., Kudo, K. \& Miyasaka, K. 2000. Physical aspects of a real-time tumor-tracking system for gated radiotherapy. Int J Radiat Oncol Biol Phys, 48, 1187-1195.

10 Tehrani, J. N., O'brien, R. T., Poulsen, P. R. \& Keall, P. 2013. Real-time estimation of prostate tumor rotation and translation with a $\mathrm{kV}$ imaging system based on an iterative closest point algorithm. Phys Med Biol, 58, 8517-8533.

Wu, J., Ruan, D., Cho, B., Sawant, A., Petersen, J., Newell, L. J., Cattell, H. \& Keall, P. J. 2011. Electromagnetic Detection and Real-Time DMLC Adaptation to Target Rotation During Radiotherapy. Int J Radiat Oncol Biol Phys, 82, e545-e553. 